\title{
CONTENIDO DE DURAMEN Y DE ALBURA EN EUCALYPTUS GLOBULUS Y ACACIA MELANOXYLON IMPLANTADAS EN ARGENTINA
}

\section{HEARTWOOD AND SAPWOOD CONTENT IN EUCALYPTUS GLOBULUS AND ACACIA MELANOXYLON GROWING IN ARGENTINA}

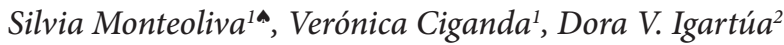

\begin{abstract}
RESUMEN
Los objetivos del trabajo fueron determinar la proporción de duramen y el ancho de la albura en Eucalyptus globulus y Acacia melanoxylon, estudiar su variación axial en el fuste y establecer relaciones entre duramen y el diámetro y volumen del fuste. Se muestrearon 10 árboles de E. globulus de 9 años y 10 árboles de A. melanoxylon de 19 a 32 años, en dos sitios de Buenos Aires, Argentina. En cuatro rodajas por árbol (base, $1.3 \mathrm{~m}, 30 \%$ y $50 \%$ de la altura total), se determinaron el área, volumen y porcentaje de duramen y el ancho de albura. En E. globulus el porcentaje de duramen disminuyó significativamente hacia el ápice ( $46 \%$ en la base y $30 \%$ en la última altura muestreada); en A. melanoxylon no hubo diferencias significativas ( $48 \%$ a $31 \%$ ). El ancho de albura fue relativamente constante a lo largo del fuste, siendo en promedio de $26 \mathrm{~mm}$ para ambas especies. El volumen de duramen con respecto al volumen del fuste fue de $37 \%$ para E. globulus y de $43 \%$ para A. melanoxylon. Se hallaron regresiones lineales significativas entre el volumen del duramen y dos variables de interés comercial e inventarial: volumen del fuste y diámetro a la altura de pecho.
\end{abstract}

Palabras clave: Área duramen, ancho albura, volumen duramen, regresiones, variación axial.

\begin{abstract}
The aims of the work were to determine heartwood and sapwood content in Eucalyptus globulus and Acacia melanoxylon trees, to study their variation and to establish relationships between heartwood and log volume and diameter. Ten trees of 9-years old E. globulus and ten trees of A. melanoxylon between 19 and 32 years old were sampled in Buenos Aires, Argentina. Heartwood area, volumes and percentage, and sapwood width were measured and calculated at four discs by tree (base, $1.3 \mathrm{~m}, 30 \%$ and $50 \%$ of total height). E. globulus heartwood percentage decreased significantly from the base upwards ( $46 \%$ at the base and $30 \%$ at the last sample height); in A. melanoxylon axial differences were not significant ( $48 \%$ to $31 \%$ ). Sapwood width was relatively constant with sample height and its means were $26 \mathrm{~mm}$ for both species. Average heartwood volume represented $37 \%$ of the log volume for E. globulus and $43 \%$ for A. melanoxylon. For both species, linear regression models show positives relationships between heartwood volume and two variables of commercial and inventorial importance: log volume and diameter at breast height.
\end{abstract}

Keywords: Heartwood area, sapwood width, heartwood volume, regressions, axial variation

\footnotetext{
${ }^{1}$ Universidad Nacional de La Plata, Facultad de Ciencias Agrarias y Forestales, CC 31, (1900) La Plata, Provincia de Buenos Aires, Argentina. ${ }^{2}$ Universidad Nacional de Mar del Plata, Facultad de Ciencias Agrarias, CC 276, (7620) Balcarce, Provincia de Buenos Aires, Argentina

^Autor de correspondencia: smonteoliva@yahoo.com.ar

Recibido: 07.10. 2011 Aceptado: 11.12.2011.
} 


\section{INTRODUCCIÓN}

En el sudeste de la provincia de Buenos Aires, Argentina, la actividad forestal ha tenido un desarrollo relativamente reciente basado en la especie Eucalyptus globulus Labill. ("eucalipto blanco") con destino a la producción de pasta celulósica kraft. Esta especie ha sido estudiada localmente en aspectos de su madera relacionados a sus usos papeleros (Igartúa y Monteoliva 2010), pero las propiedades tecnológicas asociadas a sus usos como madera sólida no han sido analizadas aún en la zona de estudio. Este recurso forestal ha sido implantado en la región desde los años 90, considerando como único destino la producción de pasta celulósica. La región cuenta además con otro recurso forestal constituido por Acacia melanoxylon $\mathrm{R}$. Br ("acacia australiana"), implantada como ornamental y como masas de protección, sin fines comerciales y no incluida aún en el mercado maderero argentino.

La región también demanda maderas para otros usos, dado el dinamismo que en los últimos años han cobrado actividades como la construcción y la industria manufacturera del mueble. Esto define una demanda de maderas de calidad para usos relacionados con estos sectores que actualmente la región no ofrece. Si bien no hay estudios que caractericen dicho mercado, puede indicarse que la demanda se atiende con madera aserrada de especies nativas y exóticas provenientes del noreste del país. Desde hace unos años se registra regionalmente cierto desabastecimiento y elevación de los precios dado que las maderas aserradas del norte del país comenzaron a participar en buena medida en la exportación cuando en los años 2002-2003 el aumento del tipo de cambio favoreció al comercio exterior de productos forestales (SAyDS 2010). Ambas especies podrían formar parte de la oferta del mercado regional de madera de calidad para usos sólidos, relacionados con las industrias de la construcción y del mueble.

Mundialmente la madera de A. melanoxylon es considerada de alto valor para la fabricación de muebles, revestimientos y ebanistería, principalmente por la calidad y atractivo color oscuro del duramen (Searle y Owen 2005, Tasmanian Timber Promotion Board 2011), claramente diferenciado de la albura en términos de color (Monteoliva et al. 2009). En la Argentina se han publicado resultados sobre evaluación de propiedades anatómicas, físicas y color de la madera de A. melanoxylon (Igartúa y Monteoliva 2009, Igartúa et al. 2009, Monteoliva et al. 2009, Monteoliva e Igartúa 2010) y se están analizando las propiedades mecánicas.

La proporción duramen/albura es un atributo de calidad de importancia para los diferentes usos finales. La transición de albura a duramen es acompañada por una serie de cambios bioquímicos. El duramen contiene extractivos y un menor contenido de humedad que la albura, mientras que ésta contiene células de parénquima vivas. Los polifenoles infiltran las paredes de las células del duramen, reduciendo la contracción y la capacidad de hinchamiento de la madera e incrementando su durabilidad y otras propiedades (Hillis 1987). Para productos sólidos, el duramen usualmente posee mayor durabilidad que la albura debido a la presencia de extractivos. El duramen es normalmente de color oscuro y frecuentemente más denso, sin embrago, no significa necesariamente una alta resistencia mecánica. El duramen generalmente tiene menor permeabilidad que la albura, esto sugiere que es mas difícil secarlo, y menos penetrable por los preservantes, pintura y colorantes (Zhang 1997). La madera destinada a productos aserrados es apreciada cuando presenta alta proporción de duramen (Knapic et al. 2006). Algo similar ocurre cuando se utiliza madera redonda descortezada para postes, disminuyendo los costos que involucra la preservación de la albura susceptible a la degradación. En el pulpado, en cambio, el impacto tecnológico de una alta proporción de duramen es negativo, es preferible una mayor proporción de albura con menores cantidades de extractivos, de esta forma se consumen menores cantidades de reactivos de impregnación y blanqueo y se obtiene mayor rendimiento (Lourenço et al. 2008). 
El volumen del duramen es acumulativo, en consecuencia la porción duraminizada del tronco se incrementa con la edad del árbol (Bradbury 2005, Searle y Owen 2005, Knapic et al. 2006, Gominho y Pereira 2000, 2005, Miranda et al. 2006). El desarrollo del duramen también está ligado al tamaño del árbol, así individuos de mayor diámetro y altura, para una misma edad, presentan mayor proporción de duramen y éste alcanza mayores alturas (Miranda et al. 2009). Esto supone que el uso de diferentes tratamientos silvícolas aplicados para aumentar los diámetros de los árboles y el volumen del rodal, afectarán positivamente la proporción de duramen. Algunos trabajos publicados para $E$. globulus lo confirman (Gominho y Pereira 2005, Miranda et al. 2009).

Algunas investigaciones han relacionado, en ambas especies, la cantidad de duramen con el diámetro a la altura del pecho y el volumen del árbol (Taylor et al. 2002, Gominho y Pereira 2000, 2005, Miranda et al. 2009). Esta caracterización puede tener importantes implicaciones en el manejo de plantaciones.

En Argentina, no hay antecedentes de estudios sobre la proporción de duramen y albura y sobre las relaciones entre duramen y variables dendrométricas para estas especies. Como se dijo antes, el sudeste de la provincia de Buenos Aires podría diversificar su producción primaria forestal hacia diferentes industrias incorporando A. melanoxylon como especie proveedora de madera aserrada de calidad, y ampliado la utilización del E. globulus, hasta el momento de uso eminentemente papelero. Para ello es necesario, entre otros aspectos, continuar la evaluación de la aptitud tecnológica de ambas especies incorporando propiedades que afectan los usos sólidos.

En el marco de la investigación en desarrollo orientada a evaluar la aptitud tecnológica de la madera de E. globulus y A. melanoxylon, los objetivos del trabajo fueron determinar la proporción de duramen y el ancho de la albura en árboles de estas especies, estudiar su variación axial en el fuste y las relaciones entre el volumen de duramen y las variables volumen del fuste y diámetro a la altura del pecho.

\section{MATERIALES Y MÉTODOS}

Se estudiaron 10 árboles de E. globulus de una plantación comercial de nueve años, de procedencia local, implantado con destino en la industria papelera. Para A. melanoxylon se estudiaron 10 árboles provenientes de rodales ubicados en dos sitios.

En el marco de investigaciones asociadas a la presente, se desarrolló la tarea inventarial que permitió establecer clases diamétricas, determinar existencias en volumen y caracterizar a las plantaciones de A. melanoxylon como de estructura de edad no uniforme.

El muestreo se realizó en base a los permisos otorgados por los propietarios de los establecimientos y a las clases establecidas por los inventarios antecedentes. Así, los árboles de ambas especies se seleccionaron al azar dentro de las cuatro clases diamétricas superiores y del estrato codominante. Investigaciones asociadas a la presente permitieron determinar la edad del material correspondiente a A. melanoxylon por procedimientos microscópicos (Monteoliva e Igartúa 2010). Este atributo (edad) no formó parte del criterio de selección del material experimental del presente trabajo.

Los 10 árboles de E. globulus y cinco de A. melanoxylon provinieron de un sitio de muestreo denominado Cinco Cerros (CC) (37 43' 59" S, 58 14' 30" O; 163 m s.n.m). Corresponde a una zona serrana, ubicada a $70 \mathrm{~km}$ del litoral, en el Partido de Balcarce, Provincia de Buenos Aires, Argentina. Los otros cinco árboles de A. melanoxylon provinieron del sitio Las Cortaderas (LC) (38 17’29” S, 
58 09’ 09” O; 31 m.s.n.m), localizado en zona de llanura a 13 km de la línea del mar, en el Partido de Gral. Alvarado, en la citada provincia.

En estas plantaciones no se practicaron intervenciones silviculturales post plantación, lo que representa la situación de ordenación forestal más característica para las plantaciones de ambas especies en la región sudeste de la provincia de Buenos Aires. Las densidades de plantación al momento del presente estudio fueron de 860 plantas/ha (E. globulus), 2013 plantas /ha (A. melanoxylon - CC) y 1656 plantas /ha (A.melanoxylon - LC)

El clima del área sudeste de la provincia es subhúmedo-húmedo, con pequeña deficiencia hídrica pero sin estación seca. Las precipitaciones medias anuales alcanzan $900 \mathrm{~mm}$, repartidas uniformemente en el año. Las temperaturas medias del mes más cálido alcanzan $20^{\circ} \mathrm{C}$ y las del mes más frío $8^{\circ} \mathrm{C}$. Las heladas invierno/primavera son frecuentes, aunque de baja intensidad (rara vez con rigores mayores a $-7^{\circ} \mathrm{C}$ ). El concepto central de los suelos regionales es el de argiudol típico (Culot 2000).

Tabla 1. Datos dendrométricos y edad de árboles selectos

\begin{tabular}{|c|c|c|c|c|c|c|}
\hline Especie & Arbol $^{*}$ & $\begin{array}{l}\text { Edad } \\
\text { (Años) }\end{array}$ & $\begin{array}{l}\text { DAP c/c } \\
(\mathrm{cm})\end{array}$ & $\begin{array}{c}\text { Altura } \\
\text { total }(\mathrm{m})\end{array}$ & $\begin{array}{l}\text { Altura comercial } \\
\text { (m) }\end{array}$ & $\begin{array}{c}\text { Altura } \\
\text { comercia } \\
\text { (\% altura } \\
\text { total) }\end{array}$ \\
\hline \multirow{10}{*}{$\begin{array}{l}\text { Eucalyptus } \\
\text { globulus }\end{array}$} & CC II 1 & 9 & 12.1 & 12.3 & 9.4 & 76 \\
\hline & CC II 2 & 9 & 11.9 & 16.4 & 12.3 & 75 \\
\hline & CC III 1 & 9 & 16.6 & 15.2 & 11.4 & 75 \\
\hline & CC III 2 & 9 & 16.6 & 12.3 & 9.3 & 76 \\
\hline & CC III 3 & 9 & 16.7 & 13.3 & 10.0 & 75 \\
\hline & CC IV 1 & 9 & 21.0 & 14.7 & 10.7 & 73 \\
\hline & CC IV 2 & 9 & 21.0 & 14.6 & 10.8 & 74 \\
\hline & CC IV 3 & 9 & 20.9 & 13.0 & 9.7 & 75 \\
\hline & CC V1 & 9 & 25.7 & 15.1 & 11.3 & 75 \\
\hline & CC V2 & 9 & 25.6 & 14.0 & 10.5 & 75 \\
\hline \multirow{10}{*}{$\begin{array}{c}\text { Acacia } \\
\text { melanoxylon }\end{array}$} & CC III & 20 & 13.2 & 12.6 & 8.1 & 64 \\
\hline & CC IV 1 & 31 & 18.5 & 12.1 & 7.7 & 63 \\
\hline & CC IV 2 & 26 & 18.2 & 13.4 & 7.2 & 54 \\
\hline & $\mathrm{CC} \mathrm{V}$ & 21 & 22.3 & 14.1 & 8.6 & 61 \\
\hline & CC VI & 21 & 25.7 & 13.3 & 6.87 & 52 \\
\hline & LC IV 1 & 27 & 16.5 & 13.6 & 6.75 & 50 \\
\hline & LC IV 2 & 26 & 15.5 & 12.6 & 9.4 & 75 \\
\hline & LC IV 3 & 32 & 16.1 & 13.5 & 8.0 & 59 \\
\hline & LC IV 4 & 25 & 16.2 & 13.7 & 7.9 & 58 \\
\hline & LC IV 5 & 19 & 17.0 & 12.9 & 7.0 & 54 \\
\hline
\end{tabular}

\footnotetext{
*Los números romanos indican clases diamétricas, las letras CC y LC indican los sitios de muestreo.
} 
Se cortaron cuatro rodajas de 3-5 cm de espesor de cada árbol, tomadas a las siguientes alturas en el fuste: base (30 cm sobre el nivel del suelo), altura de pecho ( $1.30 \mathrm{~m}$ sobre el nivel del suelo), $30 \% \mathrm{y}$ $50 \%$ de la altura total. El concepto de fuste empleado corresponde a lo indicado por Caillez (1980), lo que implicó que se considerara como su límite superior a la base de la copa. La altura del fuste fue considerada como la altura comercial. Se determinó la base de copa como el nivel donde comienzan las primeras ramas vivas de los árboles muestreados. Dicho nivel de copa para la acacia (59\% de la altura total), coincidió aproximadamente con la máxima altura muestreada, por lo tanto puede considerarse que se evaluó todo el fuste. En cambio para el eucalipto, la base de copa correspondió al nivel del $75 \%$ de la altura total, por lo tanto, existió un $25 \%$ del fuste que no fue muestreado (tabla 1 ).

Las rodajas fueron secadas al aire y pulidas en una de las superficies transversales con lijas de granulometría decreciente (80-100-120). Se realizaron dos mediciones ortogonales de los diámetros de las rodajas con corteza y sin corteza y del duramen (sentidos: norte-sur y este-oeste) con centro en la médula (figura 1). El límite del área del duramen se apreció macroscópicamente por inspección visual, siendo muy marcado en acacia y menos notorio en eucalipto.
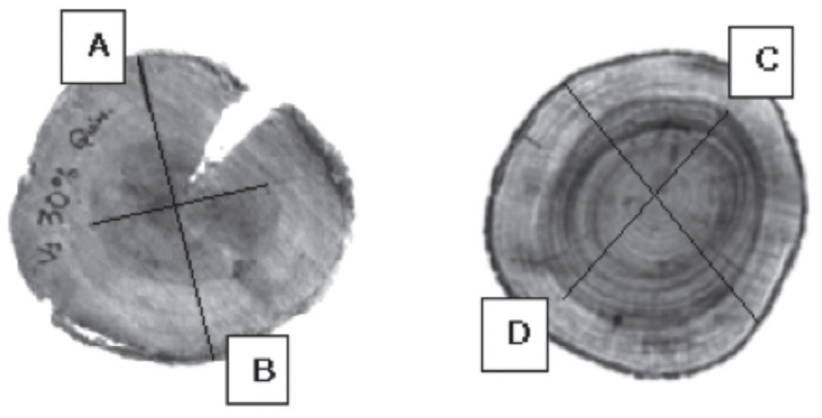

Figura 1. Fotos de una rodaja de Eucalyptus globulus (izquierda) y Acacia melanoxylon (derecha) mostrando la marcación de un diámetro sin corteza (A-B) y un diámetro de duramen (C-D)

Las áreas en la sección transversal se calcularon según las fórmulas 1 y 2, suponiendo a dichas áreas como circulares y utilizando los diámetros sin corteza:

$$
\begin{aligned}
& \mathrm{St}_{\mathrm{s} / \mathrm{c}}=\pi / 4 \cdot \mathrm{D}_{\mathrm{s} / \mathrm{c}}^{2} \\
& \mathrm{~S}_{\mathrm{HW}}=\pi / 4 \cdot \mathrm{d}^{2}
\end{aligned}
$$

Dónde:

$\mathrm{St}_{\text {s/c }}$ : área total de la sección transversal sin corteza.

$\mathrm{S}_{\mathrm{HW}}$ : área del duramen.

$\mathrm{D}_{\mathrm{s} / \mathrm{c}}$ : diámetro sin corteza, promedio de las dos orientaciones cardinales (N-S y E-O), en la sección transversal.

d: diámetro del duramen, promedio de las dos orientaciones cardinales (N-S y E-O), en la sección transversal.

Se calculó el ancho de la albura $(\mathrm{mm})$ en sentido radial por diferencia entre el diámetro promedio sin corteza y el diámetro promedio del duramen en cada rodaja. Dicha diferencia se dividió por dos. A partir de las áreas obtenidas, se calculó la proporción de duramen como porcentaje del área total sin corteza de la sección transversal, a las distintas alturas muestreadas (relación área: área). 
Los volúmenes de las trozas sin corteza y del duramen fueron calculados a partir de la fórmula de Smalian, cuya expresión es:

$$
\mathrm{V}=\left(\mathrm{g}_{\mathrm{b}}+\mathrm{g}_{\mathrm{s}}\right) \cdot \mathrm{L} / 2
$$

Donde:

V: volumen de interés a nivel de las trozas individuales (sea total sin corteza, sea de duramen, sea de albura)

$\mathrm{g}_{\mathrm{b}}$ : $\quad$ área de interés en la sección inferior o base de la troza.

$\mathrm{g}_{\mathrm{s}}$ : $\quad$ área de interés en la sección superior de la troza.

L: largo de la troza.

La suma de los volúmenes individuales de las trozas resultó en el volumen del fuste sin corteza o de duramen hasta el $50 \%$ de la altura total que, en adelante, se denominarán como "volumen del fuste" y "volumen de duramen", respectivamente El contenido de duramen también fue expresado como porcentaje del volumen del fuste sin corteza (relación volumen: volumen).

Se realizó un análisis de la varianza para evaluar a la altura de muestreo como fuente de variación en el porcentaje de duramen (área: área) y en el ancho de albura, para cada especie, en forma separada (ecuación 4). No se analizó a la edad, ni al sitio como fuentes de variación para la acacia en este estudio. Para la analizar las diferencias entre medias se utilizó la prueba de comparaciones múltiples de Tukey, con un nivel de confianza del $95 \%$.

$$
\begin{aligned}
& y_{i}=\mu+\alpha_{i}+\varepsilon_{i} \\
& i=1,2,3,4
\end{aligned}
$$

Donde:

$\mathrm{y}_{\mathrm{i}}$ : duramen o albura correspondiente a la i-ésima altura del fuste

$\alpha_{i}$ : efecto fijo de la i-ésima altura del fuste

$\varepsilon_{\mathrm{i}}$ : efecto (error) de la i-ésima altura

Se realizaron correlaciones (coeficiente de Pearson) para determinar la relación entre las variables: diámetro a la altura del pecho con corteza (DAP c/c), porcentaje duramen, volumen de duramen, volumen del fuste sin corteza. Se ajustaron regresiones lineales simples para construir modelos predictivos del volumen de duramen a partir de las variables dendrométricas DAP c/c y volumen del fuste sin corteza.

\section{RESULTADOS}

Se halló la presencia de duramen en todos los árboles, y a todas las alturas muestreadas en el fuste en ambas especies. Se observó que el perfil de distribución axial de duramen difiere entre las especies. En E. globulus se apreció un disminución del duramen del 18\% entre la altura del 30\% y altura del pecho (AP), en cambio en A. melanoxylon el descenso fue del 7\% (tabla 2). 
Tabla 2. Variación en el porcentaje de duramen (área: área) y ancho de albura en las dos especies a las distintas alturas muestreadas. Valor medio de los árboles, \pm desvío estándar. Prueba de Tukey

\begin{tabular}{|c|l|c|c|c|c|}
\hline Especie & \multicolumn{1}{|c|}{ Variables } & Base $^{*}$ & AP & $30 \%$ & $50 \%$ \\
\hline \multirow{3}{*}{$\begin{array}{c}\text { Eucalyptus } \\
\text { globulus }\end{array}$} & Duramen $(\%)$ & $46.0 \pm 9.1 \mathrm{~b}^{* *}$ & $46.7 \pm 10.8 \mathrm{~b}$ & $24.9 \pm 6.9 \mathrm{a}$ & $30.3 \pm 9.5 \mathrm{a}$ \\
\cline { 2 - 6 } & Area duramen $\left(\mathrm{cm}^{2}\right)$ & $110 \pm 40 \mathrm{~b}$ & $105 \pm 50 \mathrm{~b}$ & $31 \pm 10 \mathrm{a}$ & $15 \pm 10 \mathrm{a}$ \\
\cline { 2 - 6 } & Ancho albura $(\mathrm{mm})$ & $28 \pm 10 \mathrm{ab}$ & $25 \pm 7 \mathrm{ab}$ & $30 \pm 8 \mathrm{~b}$ & $19 \pm 5 \mathrm{a}$ \\
\hline \multirow{2}{*}{$\begin{array}{c}\text { Acacia } \\
\text { melanoxy- } \\
\text { lon }\end{array}$} & Duramen $(\%)$ & $48.7 \pm 9.1 \mathrm{~b}$ & $47.7 \pm 8.4 \mathrm{~b}$ & $40.8 \pm 10.2 \mathrm{ab}$ & $31.8 \pm 9.8 \mathrm{a}$ \\
\cline { 2 - 6 } & Área duramen $\left(\mathrm{cm}^{2}\right)$ & $130 \pm 55 \mathrm{~b}$ & $109 \pm 40 \mathrm{~b}$ & $73 \pm 25 \mathrm{ab}$ & $46 \pm 22 \mathrm{a}$ \\
\cline { 2 - 6 } & Ancho albura $(\mathrm{mm})$ & $27 \pm 9 \mathrm{a}$ & $24 \pm 7 \mathrm{a}$ & $25 \pm 8 \mathrm{a}$ & $28 \pm 8 \mathrm{a}$ \\
\hline
\end{tabular}

* Alturas de muestreo en el fuste. Base: $0.3 \mathrm{~m}$; AP: $1.3 \mathrm{~m}$; $30 \%$ y $50 \%$ : porcentajes de la altura total del fuste

${ }^{* *}$ Las letras se leen horizontalmente, letras iguales no difieren significativamente $(p<0.05)$.

Según los ANOVA, para ambas especies, la altura de muestreo fue una fuente de variación significativa $(p<0.05)$ en el porcentaje y área de duramen, manifestándose en general una disminución desde la base hasta el extremo superior del fuste (50\% de la altura total). Para E. globulus las dos alturas inferiores presentaron mayores valores que las superiores, mientras que en A. melanoxylon solo se diferenció por su menor valor la altura superior (tabla 2, prueba de Tukey).

Se observó un ancho de albura promedio (promedio simple en el fuste) de $26 \mathrm{~mm}$ para E. globulus y de $26.5 \mathrm{~mm}$ para A. melanoxylon. La altura en el fuste no resultó una fuente significativa de variación para el ancho de albura en A. melanoxylon $(\mathrm{p}=0.061)$, y sí lo fue en E. globulus $(\mathrm{p}=0.026)$ (tabla 2, prueba de Tukey).

El volumen de duramen con respecto al volumen del fuste sin corteza fue, en promedio, de $43 \%$ para A. melanoxylon y del $36 \%$ para E. globulus. Los árboles de ambas especies presentaron variación individual tanto en volumen del fuste, como en volumen de duramen (tabla 3).

Tabla 3. Edad, DAP con y sin corteza (c/c, s/c), volumen del fuste s/c y volumen de duramen (media \pm desvío estándar).

\begin{tabular}{|c|c|c|c|c|c|c|}
\hline Especie & $\begin{array}{c}\text { Edad } \\
(\mathrm{años})\end{array}$ & $\mathrm{N}$ & $\begin{array}{c}\text { DAP c/c } \\
(\mathrm{cm})\end{array}$ & $\begin{array}{c}\text { DAP s/c } \\
(\mathrm{cm})\end{array}$ & $\begin{array}{c}\text { Volumen del } \\
\text { fuste s/c }\left(\mathrm{m}^{3}\right)\end{array}$ & $\begin{array}{c}\text { Volumen de } \\
\text { duramen }\left(\mathrm{m}^{3}\right)\end{array}$ \\
\hline $\begin{array}{c}\text { Eucalyptus } \\
\text { globulus }\end{array}$ & 9 & 10 & $18.8 \pm 4.9$ & $16.14 \pm 3.9$ & $0.11 \pm 0.02$ & $0.04 \pm 0.01$ \\
\hline $\begin{array}{c}\text { Acacia mela- } \\
\text { noxylon }\end{array}$ & $19-32$ & 10 & $17.1 \pm 4.6$ & $16.41 \pm 4.7$ & $0.21 \pm 0.07$ & $0.09 \pm 0.04$ \\
\hline
\end{tabular}

Los árboles de mayores diámetros tuvieron los mayores volúmenes de duramen en ambas especies (datos no mostrados). Esta relación DAP c/c - Duramen, se apreció en todos los árboles coetáneos de E. globulus, tanto como en los de A. melanoxylon que tuvieron diferentes edades. Se observaron correlaciones positivas y significativas entre el DAP c/c y el volumen de duramen $(\mathrm{r}=0.89, p<0.05$ para A. melanoxylon $\mathrm{y} \mathrm{r}=0.91, p<0.05$ para E. globulus $)$. Del mismo modo lo fue entre el volumen del fuste sin corteza y el volumen de duramen (A. melanoxylon: $\mathrm{r}=0.94, p<0.05 \mathrm{y}$ 
E. globulus: $\mathrm{r}=0.94, p<0.05)$. La variable porcentaje de duramen se relacionó positiva y significativamente con volumen de duramen solo para A. melanoxylon $(\mathrm{r}=0.64, p<0.05)$. En cambio, no se correlacionó con el DAP, ni el volumen del fuste en ninguna de las dos especies $(p>0.05)$. El ancho de la albura no se correlacionó con el DAP ni con el volumen del fuste en ninguna de las especies $(p>0.05)$.

En ambas especies, el ajuste de modelos lineales entre las variables correlacionadas se indica en la tabla 4 .

Tabla 4. Ajuste de regresiones lineales para el volumen de duramen (Vol. D, $\mathrm{m}^{3}$ árbol ${ }^{-1}$ ) en cada especie.

\begin{tabular}{|c|l|c|c|}
\hline Especie & \multicolumn{1}{|c|}{ Ecuación de ajuste } & $\begin{array}{c}\mathrm{R}^{2} \text { ajusta- } \\
\text { do }\end{array}$ & $p$ \\
\hline $\begin{array}{c}\text { Eucalyptus globulus } \\
(\mathrm{n}=10)\end{array}$ & Vol. $\mathrm{D}=0.349$ Volumen del fuste s/c & 0.87 & $*$ \\
\cline { 2 - 4 } & Vol. $\mathrm{D}=-0.025+0.0034 \mathrm{DAP} \mathrm{c} / \mathrm{c}$ & 0.81 & $*$ \\
\hline \multirow{2}{*}{$\begin{array}{c}\text { Acacia melanoxylon } \\
(\mathrm{n}=10)\end{array}$} & Vol. $\mathrm{D}=0.588$ Volumen del fuste s/c & 0.86 & $*$ \\
\cline { 2 - 4 } & Vol. $\mathrm{D}=-0.170+0.0152 \mathrm{DAP} \mathrm{c} / \mathrm{c}$ & 0.76 & $*$ \\
\hline
\end{tabular}

\section{DISCUSION}

El material de estudio de este trabajo, que correspondió a masas sin conducción silvicultural, y, para el caso de A. melanoxylon, masas de edad no uniforme desarrolladas en base a una notable reproducción natural, mostró porcentajes y variaciones de duramen que coinciden en gran medida, con los hallados en la bibliografía.

Así, Knapic et al. (2006) analizando 20 árboles de A. melanoxylon de 30-38 años de edad y $40 \mathrm{~cm}$ de DAP, en plantaciones comerciales para madera aserrada en Portugal, publican tendencias similares de variación axial del duramen. Las muestras se obtuvieron a nueve alturas en el fuste (Base, 5, $15,35,50,65,75,85$ y $90 \%$ de la altura total), y registraron que la proporción de duramen se mantuvo estable en la parte inferior del tronco con solo un leve descenso hasta aproximadamente el $50 \%$ de la altura del árbol, decreciendo bruscamente desde allí hacia el ápice. Estas variaciones no fueron testeadas estadísticamente. A esa edad el duramen representó el $67 \%$ del área transversal del tronco en la parte inferior del árbol, y se detectó duramen hasta el $81 \%$ de la altura total, variando en los árboles individuales, entre el 67 y el 85\% de la altura total. Se observó una tendencia de disminución en la proporción del duramen en sentido axial similar a nuestros resultados, con valores de $69 \%$ (en la base) a 58\% (al 50\% de la altura total). Estos valores representan un 14-18\% más de duramen que nuestros resultados y pueden estar reflejando las diferentes condiciones de crecimiento entre esas plantaciones y las aquí estudiadas, tanto como las diferencias en tamaño de los árboles asociadas a su mayor edad.

Harrison (1974) estudió el contenido de duramen en A. melanoxylon en diferentes lugares en Sudáfrica a distintas alturas (desde la base y con intervalos de $4 \mathrm{~m}$ ) en árboles de $40 \mathrm{~cm}$ de DAP. El porcentaje de duramen promedio en la sección transversal fue de $83 \%$ y la tendencia axial fue su disminución con la altura. La diferencia de 33\% de duramen con respecto a nuestros resultados podría también estar asociada al mayor tamaño de los árboles muestreados en Sudáfrica, y que en nuestro caso presentaron DAP entre $12-25 \mathrm{~cm}$. 
Bradbury (2005) en Tasmania, en tres sitios bajo distintos sistemas silviculturales, con 210 árboles de esta especie de Acacia, de edades y DAP más similares a las de los árboles de nuestro trabajo (14-22 años y DAP 13.8-21.4 cm), halló valores de porcentaje de duramen más bajos que lo hallado en el presente (25-40\%), pero dentro de los límites del rango de datos informados para la especie.

En referencia a lo informado para E. globulus, Gominho y Pereira (2000 y 2005) analizaron 30 árboles de plantaciones comerciales para producción de celulosa, de 9 años en 3 sitios de Portugal. En esos estudios, determinaron que el duramen representó en promedio el $40 \%$ del área transversal en la base, el $31 \%, 22 \%$ y $10 \%$ en las restantes alturas analizadas $(25 \%, 35 \%$ y $55 \%$ de la altura total, respectivamente). Los valores de contenido de duramen son ligeramente inferiores a los obtenidos en el presente trabajo, pero la tendencia de variación axial resultó similar. Otros autores reportaron un patrón axial similar de la distribución del duramen en esta especie, pero en árboles de 18 años, donde el volumen de duramen representó entre el 55-65\% del volumen total del árbol (Miranda et al. 2006).

Se encontraron antecedentes bibliográficos coincidentes con los resultados aquí obtenidos en cuanto al comportamiento relativamente estable del espesor de la albura de acacia con respecto a las distintas alturas muestreadas. Knapic et al. (2006), informaron que el ancho de la albura en sentido radial en A. melanoxylon, permaneció prácticamente constante dentro del árbol con un promedio de $31 \mathrm{~mm}$ desde la parte inferior del fuste hasta el 50\% de la altura total del árbol, incrementándose a $35 \mathrm{~mm}$ en el 75\%. Miranda et al. (2009), en árboles de 18 años de E. globulus, y sobre muestras tomadas solo a la altura del pecho, determinaron un ancho promedio de albura de $18 \mathrm{~mm}$, con un rango de $15-21 \mathrm{~mm}$; y Miranda et al. (2006), en árboles de la misma edad un ancho promedio de $20 \mathrm{~mm}$. Ellos encontraron poca variación axial del ancho de la albura en las regiones más bajas del fuste, y un aumento hacia las alturas superiores, contrariamente a lo hallado en nuestro trabajo para la máxima altura muestreada (50\%). Gominho y Pereira (2005) sobre 27 árboles de esta especie, de la misma edad que los de nuestro trabajo reportaron un ancho constante de albura de $16 \mathrm{~mm}$ en promedio. Todos estos trabajos presentaron valores absolutos de ancho de albura menores a los medidos para E. globulus en nuestro estudio.

Los resultados hallados, de valores relativamente constantes de ancho de albura, soportan la teoría de que la formación del duramen ocurre como un modo de regular la cantidad de albura. Desde el punto de vista fisiológico, son necesarios adecuados volúmenes de albura, como materiales de reserva para el metabolismo y crecimiento (Taylor et al. 2002). El desarrollo del duramen está relacionado positivamente con el crecimiento en diámetro, de esta forma el árbol mantiene un ancho de albura constante en sentido radial necesario para cumplir sus funciones fisiológicas del flujo de agua. Este fenómeno es independiente de la edad cambial, pero está en relación con el crecimiento radial del árbol (Knapic et al. 2006). Nuestros resultados, analizados mediante la relación DAP/ volumen de duramen, evidenciaron que, los árboles de mayores DAP tuvieron los mayores volúmenes de duramen en ambas especies y, particularmente en A. melanoxylon esto fue así no obstante sus diferentes edades y las posibles influencias de las diferentes calidades de sitio en las que se ha desarrollado el material aquí evaluado. Esto fue indicado también por Miranda et al. (2009) en el estudio mencionado antes, quienes publicaron que los árboles de mayores diámetros contienen mayor cantidad de duramen independientemente del espaciamiento en la plantación.

Los modelos aquí ajustados entre algunas variables de inventario como predictoras (DAP con corteza y volumen del fuste) y el volumen de duramen, resultaron con un buen grado de ajuste y muestran una relación que debería ser confirmada por más evidencia empírica local, para considerarla una apropiada predicción del volumen de duramen en pie. Los autores antes mencionados también han informado correlaciones positivas entre estas variables de inventario y el volumen de duramen, en E. globulus. Gominho y Pereira (2000 y 2005), determinaron que el volumen del duramen estaba fuertemente correlacionado con el volumen del árbol $(\mathrm{r}=0.96)$ y con el DAP a través de una relación no lineal $\left(\mathrm{R}^{2}=0.95\right)$. Miranda et al. (2009) hallaron un ajuste por regresión lineal entre DAP y diámetro del duramen con $\mathrm{R}^{2}=0.77$. 


\section{CONCLUSIONES}

Ambas especies evidenciaron presencia de duramen a lo largo de su fuste hasta el $50 \%$ de la altura total, E. globulus manifestó un volumen de duramen promedio de $37 \%$ respecto a su volumen de fuste, y A. melanoxylon de $43 \%$. El contenido de duramen en las dos especies fue, en general, disminuyendo con la altura en el fuste.

Una proporción de duramen cercana a la mitad del volumen del fuste, si bien por el momento evaluada en el marco de una alta variabilidad individual, resultaría tecnológicamente apreciado para A. melanoxylon en términos de los usos sólidos de su madera. En vistas de una diversificación de los destinos industriales de E. globulus en Argentina, actualmente destinada a papel, los resultados son alentadores en cuanto al porcentaje de duramen si consideramos que el material estudiado es joven (9 años). La proporción de duramen se correlacionó con el diámetro de los árboles, por lo tanto se espera que a mayores edades, la cantidad de duramen ocupe mayor parte del volumen del fuste. Asimismo, el inicio de prácticas de manejo silvícolas que conduzcan al aumento del volumen individual en las masas regionales de estas especies permitirían esperar un aumento en la proporción del duramen.

Contrariamente, el ancho de la albura se mantuvo relativamente constante a lo largo del fuste, comportamiento más evidente en A. melanoxylon que en E. globulus. No hubo correlación entre ancho de albura y DAP o volumen comercial, con lo que podría esperarse que un aumento del tamaño de los árboles no se relacione con un aumento en la cantidad de albura, y sí de duramen. Esto puede tener importantes implicaciones en el manejo de las plantaciones regionales. Estos resultados y los de los trabajos sobre las propiedades físicas, anatómicas, químicas y mecánicas de la investigación en curso para estas especies en la zona, permiten considerar que el material implantado en el sudeste bonaerense es potencialmente proveedor de madera aserrada de calidad en ambas especies.

\section{BIBLIOGRAFIA}

Bradbury, G.J. 2005. A comparison of timber quality of Blackwood grown in young swamp forest, fence regeneration, and a plantation. Tasforests 16 (1): 95-109.

Cailliez, F. 1980. Estimación del volumen forestal y predicción del rendimiento. Volumen 1. Estudio FAO: Montes 22/1. Organización de las Naciones Unidas para la Agricultura y la Alimentación Roma. Italia. p.89.

Culot, J.P. 2000. Caracterización edafo-climática de la región sudeste de la provincia de Buenos Aires para Eucalyptus globulus. En: Primer seminario Internacional del Eucalyptus globulus en la Argentina. Mar del Plata, Argentina. Diciembre de 2000. Trabajo completo en Actas p. 2-9.

Gominho, J.; Pereira, H. 2000. Variability of heartwood content in plantation-grown Eucalyptus globulus Labill. Wood and Fiber Science 32(2): 189-195.

Gominho J.; Pereira, H. 2005. The influence of tree spacing in heartwood content in Eucalyptus globulus Labill. Wood and Fiber Science 37(4): 382-390.

Harrison, C.M. 1974. Heartwood content patterns in Acacia melanoxylon in the Southern Cape. Forestry in South Africa 15(1): 31-34.

Hillis, W.E. 1987. Heartwood and tree exudates. Springer Verlag, Berlin, 268pp. 
Igartúa, D.; Monteoliva, S.; Piter, J.C. 2009. Estudio de algunas propiedades físicas de la madera de Acacia melanoxylon en Argentina. Maderas. Ciencia y tecnología 11(1): 3-18.

Igartúa, D.V.; Monteoliva, S. 2009. Densidad básica de la madera de Acacia melanoxylon R. $\mathrm{Br}$ en relación con la altura de muestreo, el árbol y el sitio. Revista Investigación Agraria: Sistemas y Recursos Forestales 18(1): 101-110.

Igartúa D.; Monteoliva, S. 2010. Densidad básica, longitud de fibras y crecimiento en dos procedencias de Eucalyptus globulus en Argentina. Bosque 31(2):150-156.

Knapic, S.; Tavares, F.; Pereira, H. 2006. Heartwood and sapwood variation in Acacia melanoxylon R. Br. trees in Portugal. Forestry 79 (4): 371-380.

Lourenço, A; Baptista, I.; Gominho, J. 2008. The influence of heartwood on the pulping of Acacia melanoxylon wood. Journal of Wood Science 54(6): 464-469.

Miranda, I.; Gominho, J.; Lourenço, A.; Pereira, H. 2006. The influence of irrigation and fertilization on heartwood and sapwood content in 18-year-old Eucalyptus globulus trees. Canadian Journal of Forest Research 36(10): 2675-2683.

Miranda, I.; Gominho, J.; Pereira, H. 2009. Variation of heartwood and sapwood in 18-year-old Eucalyptus globulus trees grown with different spacing. Trees 23(2): 367-372.

Monteoliva, S.; Igartúa, D.V.; Matta, E.J. 2009. Color de la madera de Acacia melanoxylon implantada en Buenos Aires, Argentina. Bosque 30(3): 192-197.

Monteoliva, S.; Igartúa, D. 2010. Variación anatómica de la madera de Acacia melanoxylon implantada en el sudeste de la provincia de Buenos Aires. Rev. Fac. Agr. La Plata 109(1): 1-7.

SAyDS. Secretaría de Ambiente y Desarrollo Sustentable. 2010. Comercio Exterior de productos forestales. [En línea] <http://www.ambiente.gov.ar> [Consulta: 3 de mayo de 2010].

Searle S.D.; Owen, J.V. 2005. Variation in basis wood density and percentage heartwood in temperate Australian Acacia species. Australian Forestry 68 (1): 126-136.

Tasmanian Timber Promotion Board. 2011. Timber Research Unit, University of Tasmania. [en línea] <http://www.tastimber.tas.gov.au> [Consulta: marzo de 2011].

Taylor, A.M.; Gartner, B.L.; Morrell, J.J. 2002. Heartwood formation and natural durability. A review. Wood and Fiber Science 34(4): 587-611.

Zhang, S.Y. 1997. Wood Quality: Its Definition, Impact, and Implications for Value-Added Timber Management and End Uses. In: Zhang SY, Gosselin R, and Chauret G, Editors. Timber Management Toward Wood Quality and End-Product Value. CTIA/IUFRO International Wood Quality Workshop. Quebec, Canadá. August 18-22 1997. Part I:17-39. 
